\title{
Medical Image Analysis of Image Segmentation and Registration Techniques
}

\author{
Hemamalini G E ${ }^{\# 1}$, Dr. J Prakash ${ }^{* 2}$, \\ ${ }^{\#}$ Electronic and Communication Department, Information Science and Engineering, VTU University \\ Vemana Institute of Technology, India \\ 1'hema7412@gmail.com \\ * Bangalore Institute of Technology \\ V.V.Puram, Bangalore. India \\ 2jogaiah_prakash@gmail.com
}

\begin{abstract}
Medical Image Analysis is essential in order to detect and diagnose the various types of Cancers. In recent years there is a rise in the death rate of patients suffering from brain cancer and lung cancer. The chances of survival among people can increase if the detection is done in the earlier stage. The widely used diagnose technique is Magnetic Resonance Imaging (MRI), Computed Tomography (CT) Images which are used to present the cancer location in the brain and lungs. In this work the brain tumour and lung cancer is detected and registered through the medical images in three stages. First is the pre -processing stage, a set of medical images is filtered for removing noise by Gaussian filter, Secondly the image is segmented automatically using Otsu and KNN clustering. Edge Detection method is done by using canny detection method. Third stage is the feature extraction stage, in this stage the segmented MRI and CT images are registered to obtain the tumour. The feature detection methods used are the SIFT and SURF algorithm for both brain and lung images to obtain effective results. The SIFT and Affine Transform registration technique used increases the speed and reduces the complexity of geometrical alignments of two images that is the reference and sensed images. It also displays the minute difference between two identical images rapidly and accurately which is essential for medical diagnoses.
\end{abstract}

Keywords: Registration, SIFT, SURF and Affine Transform

\section{INTRODUCTION}

In medical science any abnormal behaviour in human being can be diagnosed prior to conformation of any disease after the diagnosis test is done. Image processing is an emerging field which assists doctors and surgeons to analyse the complex disease like cancer, brain tumour, lung cancer, breast cancer etc. Image analysis and techniques use such as MRI, CT scan X rays enable the surgeon to detect and solve problems relating to these diseases. Tumour in brain depends on their size type and location. This tumour occurs when abnormal cells from within the brain. Automatic detection of brain tumour through MRI can provide accuracy of earlier tumour detection [1]. Similarly the lung cancer is one of the most serious health problems. Early detection of lung cancer increases the survival rate of the humans. Chest radiograph and computer Tomography (CT) scan are used to detect lung cancer. CAD (Computer Aided detection) system provides an effective scanning result and increases the scanning of module detection in the lungs. For both tumour detection if done manually lot of time is consumed. So an approach to design an algorithm where the brain and lung tumour is detected automatically within less time is considered. For this purpose both CT and MRI image is used for registration and detect tumour automatically. Lowe, invents SIFT descriptor which is invariant to scale, rotation, affine transformation, noise, occlusions and is they are (1) finding scale-space extreme (2) key point localization and filtering; (3) orientation assignment; (4) key point descriptor. The first stage is to construct the key points of images by using Difference-of-Gaussian (DoG) function. H. Bay invents SURF descriptor which is invariant to a scale and in-plane rotation features. It consists of two stages such as interest point detector and interest point descriptor. SURF also extracts the key points from both the database images and the altered images. This method matches the key points between altered image and each database image. The main objective of this paper is to propose an efficient tumour detection and registration of brain and lung using SIFT and SURF methodology. The proposed method is further enhanced and results in higher accuracy of automatic detection using the effective registration technique. In this paper the tumour detection and registration of medical images are explained in four sections. Section 1 Related works 2 Proposed methodology and Steps involved. Section 3 Methodology Analysis. Section 4 Experimental Results. Section 5 Conclusion. 


\section{RELATED WORKS}

Emmanouil Skounakiser at. This research presents a new MRI diagnosis-assistive platform that, after initial creation of a "template," is capable of providing a more automatic 3-D identification of kidneys and their abnormalities (tumour, stones, and cysts). Ali Gholipouret at. Functional localization is a concept which involves the application of a sequence of geometrical and statistical image processing operations in order to define the location of brain activity or to produce functional/parametric maps with respect to the brain structure or anatomy. SIFT only describes local information and does not make use of global information. E. N. Mortensen introduced a SIFT descriptor with global context (called GSIFT), which adds a global texture vector to the basis of SIFT. H. Bay proposed SURF, which is very similar to SIFT but it adopts different processing methods in every step. Details of these different processing methods will be discussed in Section 3. H. Bay claimed that SURF is an enhanced version of SIFT. J. M. Morel proposed Affine-SIFT (called ASIFT), which follows affine transformation parameters to correct images and intends to resist strong affine issues.

In Yadav N.G proposed detection of lung module using content Based Medical Image Retrieval. Computer Aided Diagnosis System (CAD) for early detection of lung cancer from the CT [Chest computer tomography (CT) images were implemented. Region based segmentation is performed on the lung region. The features that are used in this study are texture feature. The first step in region growing is to select a set of seed points. Seed point selection is based on some user criteria i.e pixels in a certain grey scale range. The initial region begins as the exact location of these seeds. The disadvantage of this is that it cannot be applicable for multiple images for camera detectio $\mathrm{T}_{1}$ weighted $\mathrm{T}_{2}$ weighted were fused. Here the intensity, shape deformation, texture feature were extracted from each image. In [2] Disha Sharma and Gagandeep Jindal (2011) implemented $\mathrm{CAD}$ system for detection of lung cancer. For comparison between cancerous and non cancerous images texture feature were used. The most efficient method used to differentiate them is by using artificial neutral network. The main objective of this paper is to propose an efficient tumour detection and registration of brain and lung using SIFT and SURF methodology [3]. The proposed method is further enhanced and results in higher accuracy of automatic detection using the effective registration technique.

\section{PROPOSED METHODOLOGY}

The proposed method detects and registers the tumour automatically from the cerebral and lung images.

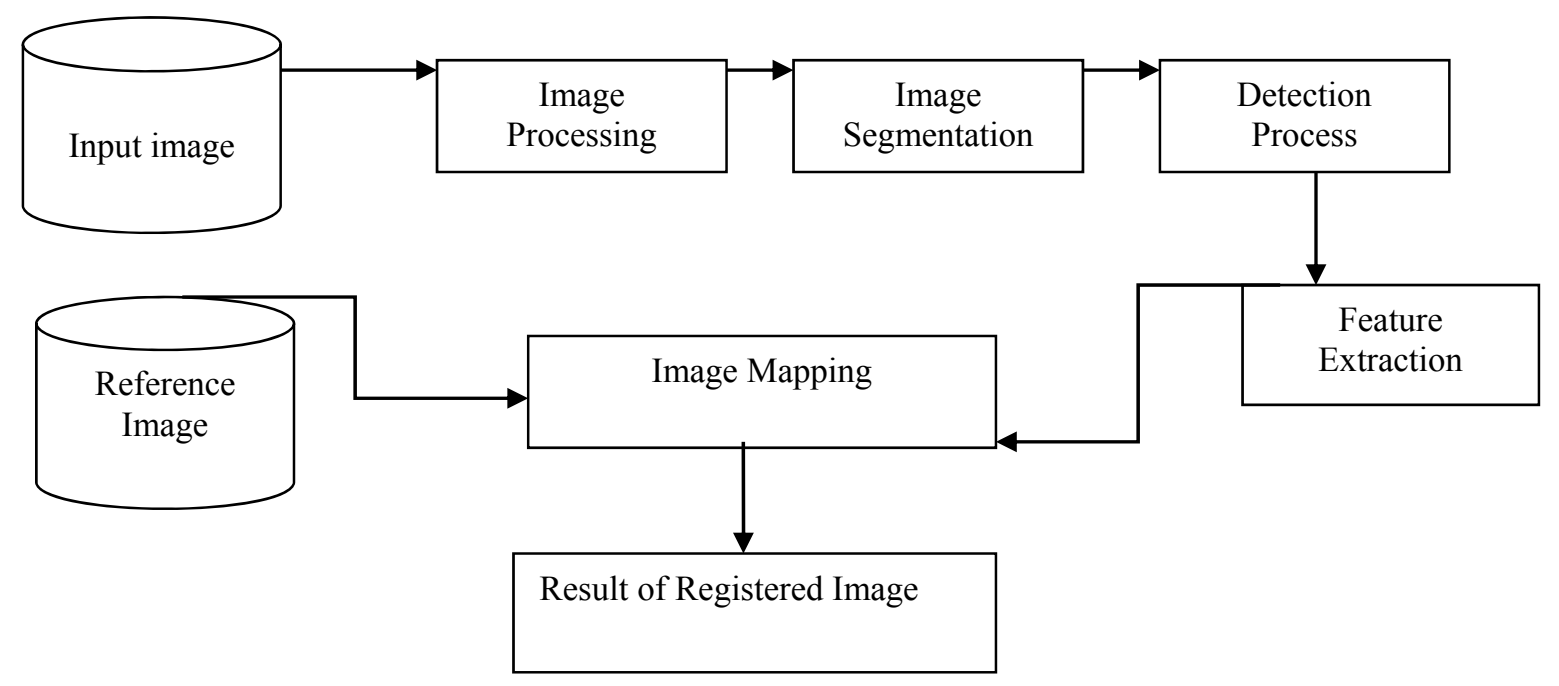

Fig 1: Block diagram of proposed methodology

\section{The steps performed are:}

1. Image of brain and lung are read and converted into matrix form.

2. Noise is removed by using Gaussian filter.

3. Segmentation technique is performed using Ostu and K-means image clustering method.

4. Edge Detection is performed using Canny detection method.

5. Feature extraction is performed using SIFT and SURF algorithm.

6. Image Mapping and Registration is done using SIFT and Affine transformation to increase the speed and reduce the complexity. 


\section{METHODOLOGY}

\section{A. Pre-processing}

In brain and lung cancer detection the main task is image pre-processing. Noise which can be induced due to various causes brings out small changes in the pixel values. This leads to false detection and therefore it is necessary to carry out image pre-processing. The blur limits the ability to see small objects, boundaries of different tissue types and other image details. Image noise is the random variation of brightness or information in images produced by the sensor and circuitry of a scanner. The common noise patterns that are present in medical images are Gaussian noise, Salt and Pepper noise and Speckle noise. In medical image processing, it is important to obtain accurate observation. Pre-processing performs filtering of noise and sharpening the edges in the image. It also converts the RGB to grey image and reshapes the image. The noise is reduced using Gaussian filter; this improves the image appearance and quality and enables easy detection of the tumour.

\section{B. Enhancement}

The contrast and intensity of the image is enhanced for the segmented process. In medical imaging enhancement is done to make the image clear for medical diagnosis. Histogram equalisation is used to enhance the contrast of the image and transform the values in the intensity image so that the histogram of the output image matches a specified histogram.

\section{Segmentation}

When an image is partitioned into multiple segments (set of pixels) it is called Image Segmentation. Segmentation represents the image into a simplified version so that it is easier to analyse. It is also used to locate objects and boundaries in images. In medical imaging segmentation enables in study of anatomical structure, locate tumour and detect abnormalities. It provides information for doctors which are useful in treatment planning. The simplest method of image segmentation is known as the thresholding method. This method is based on thresholds values into binary image. In this work OTSU and K-means clustering method is used for image segmentation [4]. K-means clustering aims to partition $n$ observations into $k$ clusters in which each observation belongs to the cluster with the nearest mean, serving as a prototype of the cluster. This results in a partitioning of the data space into Voronoi cells. Given a set of observations $(\mathrm{x} 1, \mathrm{x} 2, \ldots, \mathrm{x} n)$, where each observation is a $d$-dimensional real vector, $k$-means clustering aims to partition the $n$ observations into $k$ sets $(k$ $\leq n) \mathrm{S}=\{S 1, S 2, \ldots, S k\}$ so as to minimize the within cluster sum of squares (WCSS).

$$
\arg \min \sum_{x j \in S i}^{i} \quad \sum_{i=1}^{k}\|\mathrm{xj}-\mu \mathrm{i}\| \ldots
$$

where $\mu i$ is the mean of points in $S i$.

$\mathrm{K}$ means algorithm starts clustering by assigning $\mathrm{k}$ initial central points, either randomly or using heuristic data. It then groups each image pixel under the central point which it is closest to. Next, it calculates new central points by taking average of the pixels grouped under each central point. The two former algorithmic steps are repeated alternately until convergence.

\section{Feature Extraction}

The feature extraction of the images are performed using the SIFT and SURF algorithm. The SIFT (Scale Invariant Feature Transform) [5] is an approach for extracting distinctive invariant features from images. It is widely used in image matching. SIFT descriptor detects the extreme points through the whole scale space. SURF (Speeded-Up Robust Features)[6] is an improvement of SIFT with the same method of calculating the sum of response belonging to the interest point' neighbours, SIFT sums up the gradients and SURF sums up the wavelet responses and is applied in interest point selection. To obtain the high speed in real time calculation and more robust to rotation both these methods are integrated. The distinctive features from images are extracted from distinctive locations from the images such as edges, blobs etc [7]. Keypoint detectors should be highly repeatable. Then the neighbourhood regions are picked around every keypoint and distinctive feature descriptors are computed from each region. As such SIFT and SURF descriptors are reliable due to the efficient performance and are used in many applications.

\section{E. SIFT algorithm}

The SIFT algorithm has four main steps:

- Scale Space Extrema Detection

- Key point Localization

- Orientation Assignment 
- Description Generation.

1) Scale Space Extrema Detection- is to identify location and scales of key points using scale space extrema in the DoG (Difference-of-Gaussian) functions with different values of $\sigma$, the DoG function is convolved of image in scale space separated by a constant factor $\mathrm{k}$ as in the following equation.

$$
D(x, y, \sigma)=(G(x, y, k \sigma)-G(x, y, \sigma) \times I(x, y) \ldots
$$

Where, $\mathrm{G}$ is the Gaussian function and $\mathrm{I}$ is the image. Now the Gaussian images are subtracted to produce a DoG, after that the Gaussian image subsample by factor 2 and produce DoG for sampled image. A pixel compared of $3 \times 3$ neighborhood to detect the local maxima and minima of $\mathrm{D}(\mathrm{x}, \mathrm{y}, \sigma)$. Scale-space extrema detection, double size (width $*=2$, height $*=2$, size $\left.{ }^{*}=4\right)$ and use scale-space extrema in the difference-of-Gaussian function convolved with image pre-smooth the image with the Gaussian function[8].

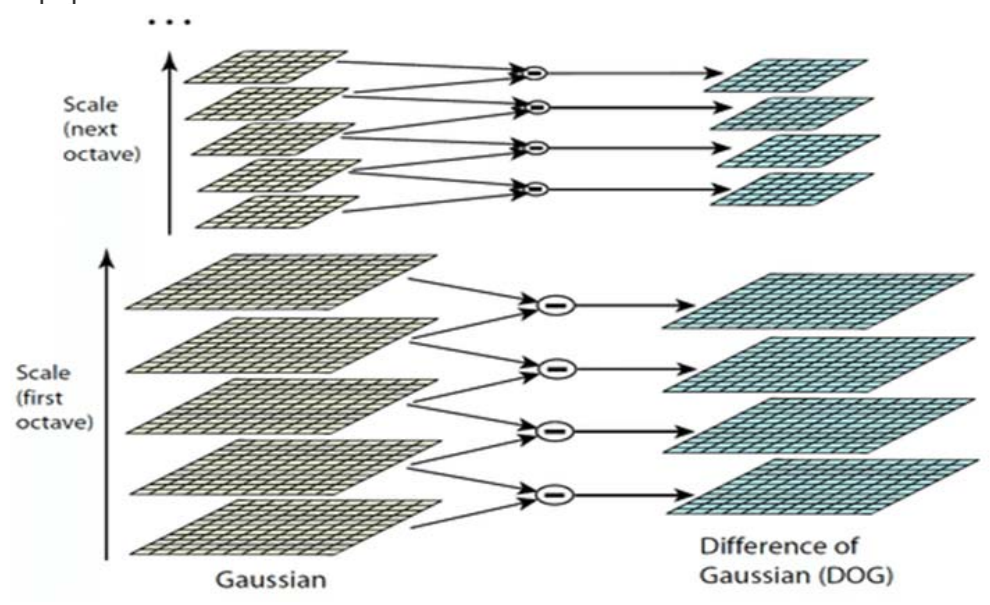

Fig 2: Difference-of-Gaussian

2) Key point localization - key point candidates are localized and refined by eliminating the key points where they rejected the low contrast points[9]. This is performed by rejecting those keypoints, which have low contrast or are poorly localized along an edge. Unstable extremas are detected by considering a threshold over the extremum of the Taylor expansion of $D(x, y$,

3) Orientation assignment - the orientation of key point is obtained based on local image gradient. Orientation to each feature based on local image gradient. The gradient's absolute value and direction are given by Figure 1 illustrates the computation of the key point descriptor [10]. First the image gradient magnitudes and orientations are sampled around the key point location, using the scale of the key point to select the level of Gaussian blur for the image. In order to achieve orientation invariance, the coordinates of the descriptor, then the gradient orientations are rotated relative to the key point orientation. Figure 1 illustrated with small arrows at each sample location on the left side.
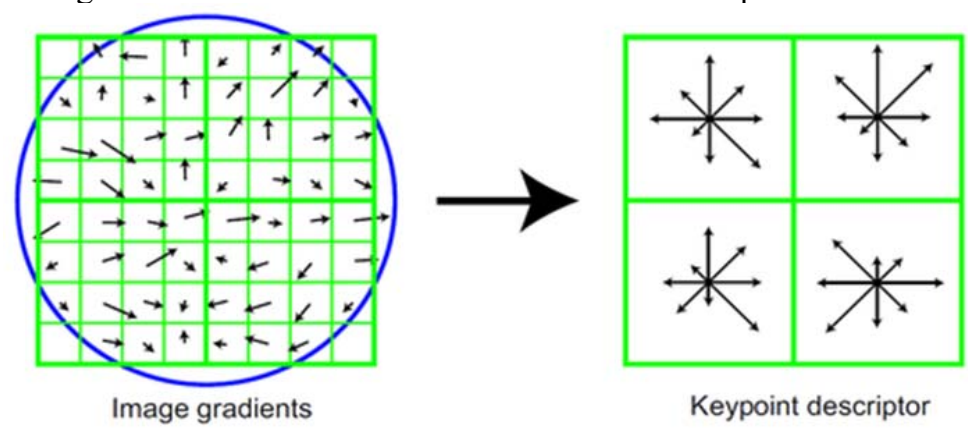

Fig 3: SIFT Descriptor Generation

4) Key point descriptor- is shown on the right side of Figure 3. It allows for significant shift in gradient positions by creating orientation histograms over $4 \times 4$ sample regions. The figure shows 8 directions for each orientation histogram with the length of each arrow corresponding to the magnitude of that 
histogram entry[11]. A gradient sample on the left can shift up to 4 sample positions while still contributing to the same histogram on the right. So, $4 \times 4$ array location grid and 8 orientation bins in each sample. That is 128 -element dimension of key point descriptor.

\section{F. SURF Algorithm}

SURF (Speed Up Robust Features) algorithm, is base on multi-scale space theory and the feature detector is base on Hessian matrix. Since Hessian matrix has good performance and accuracy. In image $I, x=(x, y)$ is the given point, the Hessian matrix $\mathrm{H}(\mathrm{x}, \sigma)$ in $\mathrm{x}$ at scale $\sigma$, it can be define as

$$
\mathbf{H}(\mathbf{x}, \boldsymbol{\sigma})=\left[\begin{array}{ll}
\operatorname{Lxx}(\mathrm{X}, \sigma) & \operatorname{Lxy}(\mathrm{X}, \sigma) \\
\operatorname{Lyx}(\mathrm{X}, \sigma) & \operatorname{Lyy}(\mathrm{X}, \sigma)
\end{array}\right]
$$

Where $\operatorname{Lxx}(\mathbf{x}, \sigma)$ is the convolution result of the second order derivative of Gaussian filters $\partial^{2} \partial x^{2}(\sigma)$ with the image I in point $\mathbf{x}$, and similarly for Lxy $(\mathbf{x}, \sigma)$ and Lyy $(\mathbf{x}, \sigma)$.SURF creates a "stack" without 2:1 down sampling for higher levels in the pyramid resulting in images of the same resolution. Due to the use of integral images[12], SURF filters the stack using a box filter approximation of second-order Gaussian partial derivatives. Since integral images allow the computation of rectangular box filters in near constant time. In Fig 4 shows the Gaussian second orders partial derivatives in y-direction and xy- direction.

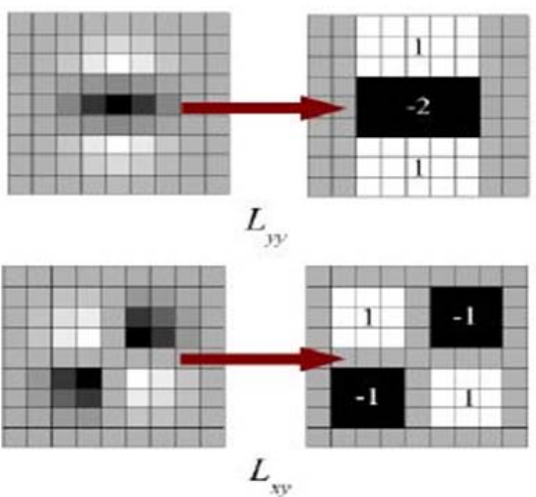

Fig 4: The Gaussian second orders partial derivatives

The Gaussian second orders partial derivatives in y-direction and xy-direction [14]. In descriptors, SIFT is good performance compare to other descriptors. The proposed SURF descriptor is based on similar properties. The first step consists of fixing a reproducible orientation based on information from a circular region around the interest point. And second construct a square region aligned to the selected orientation, and extract the SURF descriptor from it. In order to be invariant to rotation, it calculate the Haar-wavelet responses in $\mathrm{x}$ and $\mathrm{y}$ direction.

\section{G. Image Matching and Registration}

SIFT is an image local feature (extractor) description algorithm and has strong matching ability. Image matching is based on global feature and local feature based matching algothrim[15]. Local feature based matching algorithm is local feature based algorithm. Local feature based has two stages such as interest point detection and description. A good local feature detection and descriptor has high speed feature detection and capability to achieve quick matching with respect to illumination and rotation. Feature descriptors are extracted from sample images and stored.

The feature descriptors of a query image are then matched with all trained image features and the trained image giving maximum correspondence is considered the best match. Image registration is defined as the process of mapping the input images with the help of reference image. Affine image registration is used greatly in many fields such as medical imaging, remote sensing, computer vision and in medical research. Monomodal registration is registering images taken by the same imaging modality/sensor (MRI, CT, etc.)[16]. Fourier domain based cross-correlation methods are used often for monomodal image registration. Feature based methods of registration are preferred such as linear transformation and non-rigid transformation. Affine transform is a linear transform which maps pixel coordinates into new coordinates by applying a linear combination of rotation, scaling, shearing, and translation matrices. The affine transformation preserves the co linearity relation between the points, that is point which lie on same line continue to be collinear after the 
transformation. SIFT algorithm with matching algorithm based on affine transformation is implemented[17]. The feature point improves the robust of registration algorithm greatly.

\section{EXPERIMENTAL RESULT}

Two images are considered such as the brain input image and the reference image for the purpose of experiment. Features are detected in the image using the SIFT and SURF algorithm. The proposed algorithm is used to detect and locate the tumour or the position of the abnormal regions in the MIR image of brain and lungs. The result obtained is important in the diagnosis. Registration is performed on the images of brain and lung. Detected images of each respective set of images has been registered using the SIFT and Affine Transformation. The results of each image are shown below.

\section{A. Brain Images}
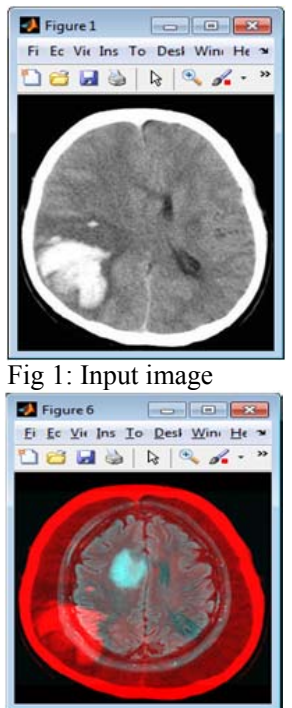

Fig 4: Image mapping

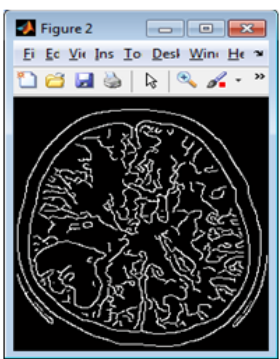

Fig 2: canny detection

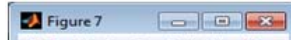

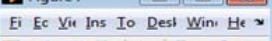

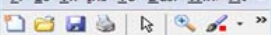

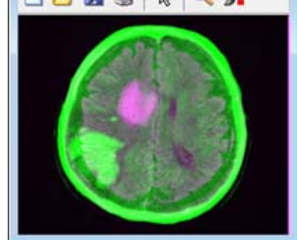

Fig 5: Image registrations

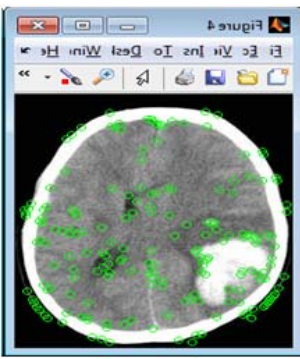

Fig 3: Feature point detection

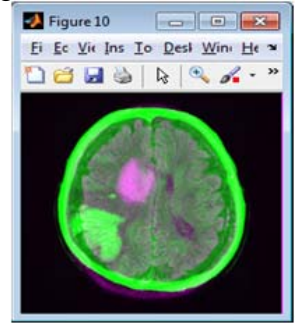

Fig 6: Affine transformation

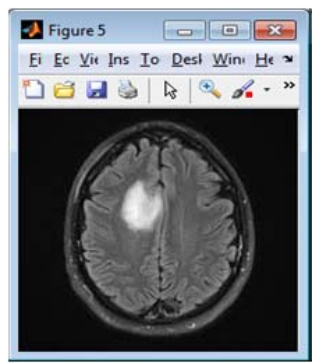

Fig 4: Reference image

Digure 11 a Ei Ec Vir Ins Io Desi Wini ㅂe v

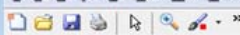

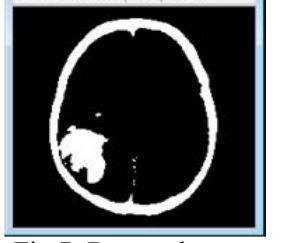

Fig 7: Detected tumour

\section{B. Lung Images}

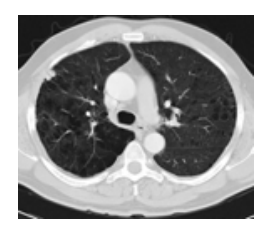

Fig 1: Input image

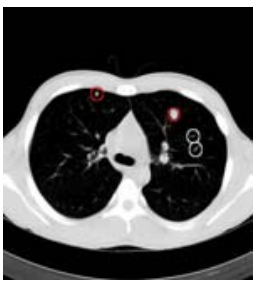

Fig 5: Reference image

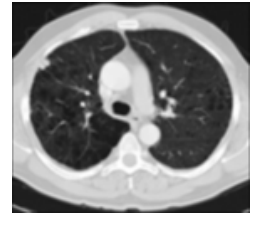

Fig 2: Rgb to bW

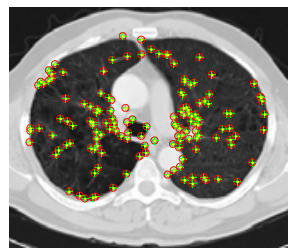

Fig 6: SIFT Detection

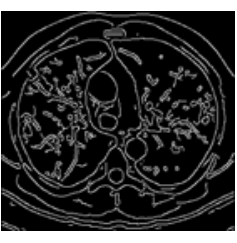

Fig 3: Edge detection
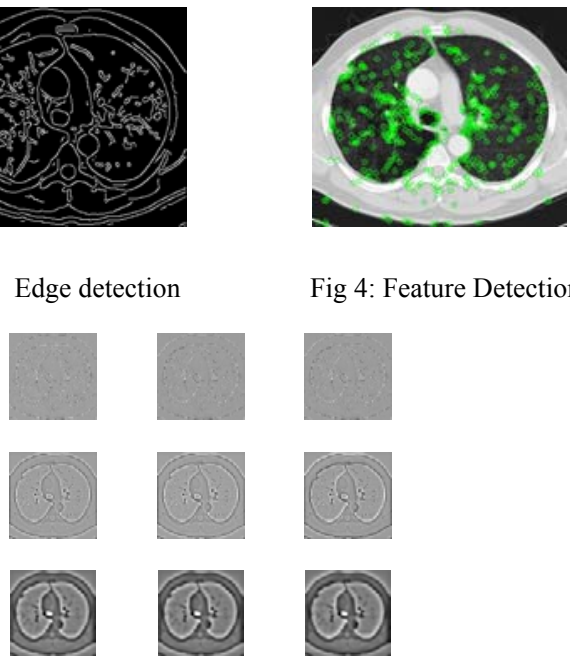

Fig 4: Feature Detection
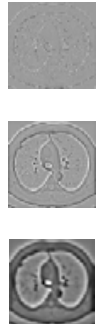

Fig 7: Image detection \& matching 


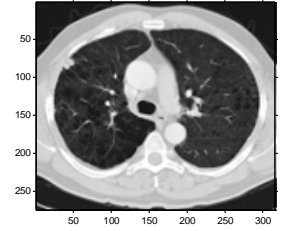

Fig 8: Imageregistration

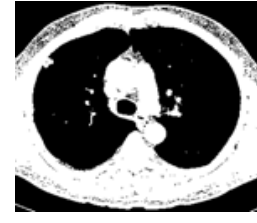

Fig 9:Tumour Detection

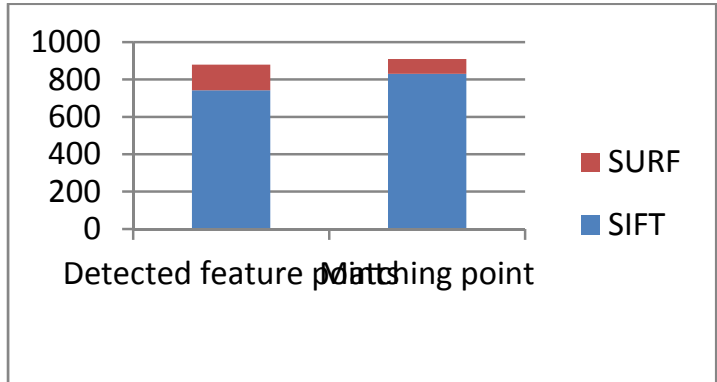

Fig10: SIFT and SURF algorithm

Table1: SIFT and SURF Algorithm

\begin{tabular}{|l|c|c|}
\hline Feature Points & $\begin{array}{c}\text { SIFT (Scale Invariant } \\
\text { Feature Transform) }\end{array}$ & $\begin{array}{c}\text { SURF (Speed Up } \\
\text { Robust Feature) }\end{array}$ \\
\hline Detected feature Points & 742 & 831 \\
\hline Matching feature point & 138 & 79 \\
\hline Feature matching Time & $0.769 \mathrm{~s}$ & $0.567 \mathrm{~s}$ \\
\hline
\end{tabular}

From the above Fig 1 is the input tumour image. De-noising of the image is done using Gaussian filter. This filter suppresses the noise and it is smoothed out. Edge detection is performed by using canny detection as shown in Fig 2. SIFT and SURF algorithm is performed for feature point detection presented in Fig 3 . The reference image is considered in Fig 4. Fig 5, 6 shows the image mapping and registration between the input and reference image using SIFT and Affine Transformation. The registered detected tumour image is presented in Fig 7. Similarly the same method is carried out for the lung images. It is shown in Fig 1- 9. The experimental results are shown in table 1 . Here the detected feature points, matching points and feature matching time are estimated for both SIFT and SURF algorithm.

\section{CONCLUSION}

This paper focuses on the tumour detection and registration of brain and lung images. The proposed SIFT and SURF algorithm shows effective method for segmentation of brain and lung tumour from the two dimensional MRI and CT images. The proposed registration methods, SIFT and Affine Transformation results in efficient matching and complexity time. It also represents and displays minute difference and gives more accurate result automatically in very less period of time. The matching feature points and feature matching time 
of SIFT and SURF are considered and from the results it is concluded that the SURF features perform slightly better than SIFT. SURF algorithm proves to be more efficient and robust and enhances the SIFT method.

\section{REFERENCE}

[1] Y. Li, L. Liu, L. Wang, D. Li and M. Zhang, "Fast SIFT Algorithm based on Sobel Edge Detector, Consumer Electronics, Communications and Networks (CECNet)", (2012), pp. 1820-1823.

[2] T. Chaves, L. Figueiredo, A. Da Gama, C. de Araújo and V. Teichrieb, "Human Body Motion and Gestures Recognition Based on Checkpoints", 14th Symposium on Virtual and Augmented Reality, (2012), pp. 271-278.

[3] F. Y. Qing, S. B. Lin and W. J. Fang, “An improved SIFT algorithm”, Journal of Harbin Engineering University, vol. 31, (2010), pp. 560-564.

[4] M. Wen and C. Zhong, "Application of Sobel Algorithm in Edge Detection of Image", China High-tech Enterprise, (2008), pp. 57-62.

[5] Z. Hou, L. Zhao, L. Gu, G. Lv and M. Wang, “An improved Image Registration Method Based on SIFT”, 2009 International Asia Symposium on Intelligent Interaction and Affective Computing, (2009), pp. 87-90

[6] Evans, Notes on the OpenSURF Library, University of Bristol (UK), 2009. Available from http://www.chrisevansdev.com. A. Moore, An introductory tutorial on kd trees, Technical Report No. 209, University of Cambridge, 1991.

[7] N. Khan, Indoor Environmental Images (Computer Science Department), Otago University (NZ), 2010.

[8] A. Efros, M. Hebert and T. Kanade, Image Matching in Large Scale Indoor Environment, Proc. International Conference on Computer Vision and Pattern Recognition Workshop, pp. 33-40, 2009. Available from http://www. hwkang.com/.

[9] Ouyang, W., Tombari, F., Mattoccia, S., Di Stefano, L.,Cham, W.-K. (2012). Performance evaluation of full search equivalent pattern matching algorithms. IEEE Transactions on Pattern Analysis and Machine Intelligence, 34 (1), $127-143$.

[10] Birinci, M., Diaz-de-Maria, F., Abdollahian, G. (2011). Neighborhood matching for object recognition algorithms based on local image features. In IEEE Digital Signal Processing Workshop and IEEE Signal Processing Education Workshop (DSP/SPE), 4-7 January 2011. IEEE, 157-162.

[11] Mian, A., Bennamoun, M., Owens, R. (2010). On the repeatability and quality of keypoints for local featurebased 3D object retrieval from cluttered scenes.International Journal of Computer Vision, 89 (2-3), 348-361.

[12] Seok-Wun Ha, Yong-Ho Moon, "Multiple Object Tracking Using SIFT Features and Location Matching" ,International Journal of Smart Home Vol. 5, No. 4,pp. 17-26, October 2011.

[13] Georgiadis. Et all (2008), 'Improving Lung Cancer characterization on CT by probabilistic neural networks and non-linear transformation of textural features', Computer Methods and program in biomedicine, Vol 89, pp24-32.

[14] Gile N., Wang Ning, Nathalie C.K., Siewe, F., Lin Xudong and Xu De(2008), 'A case study of image retrieval on lung cancer chest Xray pictures', Signal Processing, 9th International Conference on, Vol., no., pp.924, 927, 26-29.

[15] Dignam J.J., Huang L., Ries L., Reichman M., Mariotto A. and Feuer E. (2009), 'Estimating cancer statistic and other-cause mortality in clinical trial and population-based cancer registry cohorts', Cancer 10.

[16] Leyla Zhuhadar, IEEE Member and Gopi Chand Nutakki, IEEE Member, University of Louisville, Louisville, USA: Hybrid Appearance Based Disease Recognition of Human Brains, International Conference on Information Visualisation, 2012.

[17] J. Samuel, M. Dong, J. Hua, and E. M. Haacke: Wayne State University, Detroit, MI, United States: Brain Tumor Detection Using Scale Invariant Feature Transform, 2011

\section{AUTHOR PROFILE}

Author1 Name Hemamalini G.E obtained her B.E degree in Electrical and Electronics and Engineering from U.V.C.E, Karnataka, India, in 1999, M.tech degree Visvesvaraya Technological University, India in 2008. She is currently working as Assistant Professor in the department of Electronics and Communication and Engineering at Vemana Institute of Technology,Bangalore. She is pursuing her Ph.D at Visvesvaraya Technological University, India in the area of Image Fusion under the guidance of Dr.J.Prakash, Professor and Head, Department of Information Science and Engineering, Bangalore Institute of Technology, Bangalore.

Author2 Name Dr. J. Prakash received the B.E degree in Computer Science and Engineering from Mysore University, Karnataka, India, in 1989, M.S degree in IT from University of Indore, Madhya Pradesh, India in 1995 and Ph.D from Dr. MGR Educational and Research Institute, University, Chennai, India in 2010. From 1989 to 1996 he worked as lecturer in the department of Computer Science and Engineering at Adichunchanagiri Institute of Technology. Chikmagalur, Karnataka, India. From 1997 to 2010 he worked as Assistant Professor of Information Science and Engineering, at Bangalore. 